\title{
AVALIAÇÃO FUNCIONAL DO MÚSCULO TRAPÉZIO E NERVO ESPINHAL PÓS-ESVAZIAMENTO CERVICAL ATRAVÉS DA ELETRONEUROMIOGRAFIA - ESTUDO DE 25 PACIENTES
}

\section{FUNCTIONAL EVALUATION OF TRAPEZIUS MUSCLE AND SPINAL NERVE AFTER NECK DISSECTION THROUGH ELETRO-NEUROMIOGRAPHY (ENM) - STUDY OF 25 PATIENTS}

\author{
José Carlos de Oliveira ${ }^{1}$ \\ Maria Paula Curado ${ }^{1}$ \\ Márcio Roberto Barbosa Silva ${ }^{2}$ \\ Sueli Mitiko Gomi Kuwae ${ }^{2}$ \\ Ricardo Pires de Souza $^{3}$ \\ Abrão Rapoport, TCBC-SP ${ }^{4}$
}

\begin{abstract}
RESUMO: Objetivo: Foram estudados 25 pacientes portadores de neoplasias malignas da cabeça e pescoço (20 de vias aerodigestivas superiores e cinco da glândula tireóide), submetidos a esvaziamentos cervicais uni ou bilaterais (33 procedimentos), sendo 15 supra-omohióideos, 11 funcionais e sete em campos alargados Método: Através da eletroneuromiografia (ENM), foram avaliados funcionalmente o músculo trapézio e o nervo espinhal após os diferentes procedimentos, aos 30 e 180 dias. Resultados: Foram aferidos para as três formas de linfadenectomia $94 \%$ de desnervação do músculo trapézio, severa em $68 \%$ e moderada $32 \%$ (p = 0,001), portanto valores significativos. Quanto à avaliação do tipo de lesão do nervo espinhal, após 30 dias observou-se lesão de axônio (axonotmese) em 31 dos 33 procedimentos. Com relação à reinervação, esta foi detectada após 180 dias, sendo boa $(21 \%)$, moderada $(72 \%)$ e ruim $(7 \%)$ para valores de $\mathrm{p}=0,001$ de significância estatística. Conclusões: A eletroneuromiografia foi um método efetivo na avaliação da unidade neuromuscular e o tipo de esvaziamento cervical conservador não foi determinante de alterações destas estruturas.
\end{abstract}

Descritores: Músculo trapézio; Nervo espinhal; Esvaziamento cervical; Eletroneuromiografia.

\section{INTRODUÇÃO}

Após a padronização terapêutica radical através dos esvaziamentos cervicais para linfonodos metastáticos ou não em $1906^{1}$, observou-se que a lesão do nervo espinhal e a atrofia do músculo trapézio caracterizavam um grande desconforto pós-operatório chamado síndrome do ombro ${ }^{2,3}$. A partir deste fato, procuraram-se alternativas conservadoras da integridade do nervo, ou enxerto nervoso ou neu- rorrafia nervosa primária, todas contemplando a radicalidade oncológica, impedindo a queda anatômica do ombro e incapacidade funcional de abdução e elevação do braço ${ }^{4-6}$. Daí surgem os esvaziamentos mais conservadores e modificados, acompanhados de fisioterapia com exercícios de reabilitação, conservação do plexo cervical superficial. Para avaliar o grau de dano funcional destas estruturas, a eletroneuromiografia (ENM), descrita por Galvani ${ }^{7}$ em 1791, possibilitou a pesquisa do comprometimento de unidades

1. Mestrando do C.P.G. Hospital Heliópolis, Hosphel, São Paulo.

2. Eletrofisiologista Clínico do Hospital Araújo Jorge, Goiânia, Goiás.

3. Mestre em Cirurgia de Cabeça e Pescoço pelo Hospital Heliópolis, Hosphel, São Paulo.

4. Coordenador do Curso de Pós-Graduação em Cirurgia de Cabeça e Pescoço do Hospital Heliópolis, Hosphel, São Paulo.

Recebido em 31/10/2000.

Aceito para publicação em 31/07/2001.

Trabalho realizado no Serviço de Cirurgia de Cabeça e Pescoço do Hospital Araújo Jorge, Goiânia, Goiás 
motoras ou de qualquer um de seus elementos, que vão desde a célula do corno anterior às raízes nervosas e nervos periféricos. Para avaliação da lesão do nervo periférico, Seddon ${ }^{8}$ em 1943 classificou as alterações estruturais nervosas em função do grau de ruptura estrutural da bainha de mielina (célula de Schwan), do axônio e do corpo celular. Assim, objetivamos avaliar as alterações motoras do músculo trapézio e a sua reinervação pós-desnervação parcial por lesão do nervo espinhal após esvaziamentos cervicais funcional, supra-omohióideo e em campo alargado através da eletroneuromiografia, visto que a exposição da estrutura nervosa, para sua conservação, sem secção, implica manobras cirúrgicas de tração e desvascularização, com conseqüente desnervação do músculo trapézio.

\section{MÉTODO}

Estudamos prospectivamente 25 pacientes portadores de neoplasias malignas da cabeça e pescoço, submetidos a 25 esvaziamentos cervicais unilaterais e oito bilate- rais, no Hospital Araújo Jorge, Goiânia, Goiás, de junho de 1998 a dezembro de 1999. Destes, 17 (68,0\%) eram homens e oito (32\%) mulheres. Quanto à histopatologia, 20 (80\%) eram carcinomas epidermóides (13 de andar inferior da boca, três de laringe, um de palato fibroso, um de área retromolar, um de loja amigdalina e um da hipofaringe) e cinco $(20,0 \%)$ carcinomas diferenciados de tireóide (três papilíferos, um folicular e um medular). Foram classificados pelo TNM da UICC/AJC de 1997, estando distribuídas as lesões epidermóides no estádio I (dois pacientes), II (três pacientes), III (cinco pacientes) e IV (cinco pacientes) e as neoplasias de tireóide no estádio III (cinco casos).

Quanto ao procedimento cirúrgico, estes foram grupados conforme a Tabela 1 .

Em todos os 33 procedimentos (15 supra-omohióideos, 11 funcionais e sete em campo alargado), o músculo trapézio teve sua inervação (nervo espinhal) avaliada, visto que a integridade da estrutura nervosa é prejudicada por manobras de tração e desvascularização, determinando por vezes desnervação muscular parcial e, conseqüentemente, níveis de reinervação de curto prazo (30 dias) e de longo prazo (180 dias), após tratamento cirúrgico. Nos esvaziamentos

Tabela 1

Relação de pacientes submetidos a esvaziamento cervical associado ou não à radioterapia

\begin{tabular}{|c|c|c|c|}
\hline Caso & Paciente & Tipo de Esvaziamento Cervical & $R x T$ \\
\hline 1 & R.B.S & E.C.F.D. & - \\
\hline 2 & C.N.F. & E.C.F.D. & - \\
\hline 3 & J.F.S.F. & E.C.F.D. \& E.C.F.D. & RxT \\
\hline 4 & S.V.B. & E.C.I.II.II.D. & $\mathrm{RxT}$ \\
\hline 5 & G.J.F.S. & E.C.F.E. & $\mathrm{RxT}$ \\
\hline 6 & A.L.S. & E.C.F.D. & $\mathrm{RxT}$ \\
\hline 7 & J.C.M. & E.C.I.II.III.E. & $\mathrm{RxT}$ \\
\hline 8 & A.T. & E.C.I.II.III. & RxT \\
\hline 9 & T.S.S. & E.C.I.II.III.E \& E.C.I.II.III.D. & - \\
\hline 10 & J.R. & E.C.I.II.III.E \& E.C.I.II.III.D. & $\mathrm{RxT}$ \\
\hline 11 & J.P.M. & E.C.I.II.III.E. & - \\
\hline 12 & R.M.R. & E.C.F.E. & - \\
\hline 13 & W.C.B. & E.C.I.II.III.D. & - \\
\hline 14 & E.C. & E.C.I.II.III.D. & RxT \\
\hline 15 & A.F.C. & E.C.I.II.III.E \& .C.II.III.IV.D. & - \\
\hline 16 & V.E.A. & E.C.F.D.+E.C.I.II.III.E. & $\mathrm{RxT}$ \\
\hline 17 & R.C.F. & E.C.II.III.IV.E. \& E.C.II.III.IV.D. & - \\
\hline 18 & A.L.C. & E.C.II.III.IV.E. \& E.C.II.III.IV.D. & - \\
\hline 19 & V.B.S. & E.C.F.D. & - \\
\hline 20 & I.M.M. & E.C.I.II.III.E. & $\mathrm{RxT}$ \\
\hline 21 & L.B. & E.C.F.D. & RxT \\
\hline 22 & J.L.S. & E.C.I.II.III.D. & - \\
\hline 23 & C.S.C & E.C.F.D. & - \\
\hline 24 & J.R.A. & E.C.I.II.III.D. & - \\
\hline 25 & M.A.J. & E.C.I.II.III.E \& E.C.I.II.III.D. & RxT \\
\hline
\end{tabular}

E.C.I.II.III.E. - Esvaziamento Cervical Supra-omohióideo Esquerdo

E.C.I.II.III.D. - Esvaziamento Cervical Supra-omohióideo Direito

E.C.F.E. - Esvaziamento Cervical Funcional Esquerdo

E.C.F.D. - Esvaziamento Cervical Funcional Direito

E.C.II.III.IV.D. - Esvaziamento Cervical em Campo Alargado Direito

E.C.II.III.IV.E. - Esvaziamento Cervical em Campo Alargado Esquerdo

RxT - Radioterapia 
cervicais unilaterais, comparando-se o lado operado com o não operado, e nos bilaterais, a aferição foi no pré e pósoperatório (30 e 180 dias após o ato cirúrgico).

Quanto ao tratamento radioterápico, este foi indicado em 15 pacientes (dez com lesão de vias aerodigestivas superiores e cinco de tireóide) na dose de 5 a 6.000cGy.

Foi utilizado o eletromiógrafo uni ou bilateralmente, Neuropack four mini, de marca Nihon Kohden, sendo o exame dividido em duas etapas:

\section{Etapa 1}

Após estímulo do nervo espinhal utilizando-se eletrodo de captação de superfície sobre o músculo trapézio em sua porção superior (a $5 \mathrm{~cm}$ do processo espinhoso de $\mathrm{C}_{7}$, ponto de referência para o estímulo, aplicado de 1 a $2 \mathrm{~cm}$ posteriormente ao lado do músculo esternocleidomastóideo, no nível da margem superior da cartilagem tireóide), avaliou-se a velocidade de condução nervosa. Quanto à calibração do aparelho, utilizou-se freqüência de $8 \mathrm{~Hz}$ a $8 \mathrm{kHz}$, com varredura de $2 \mathrm{msec} /$ div e latência de 1,8 a $3,0 \mathrm{mseg}$.

\section{Etapa 2}

Aferiu as porções média e superior do músculo trapézio com eletrodos de agulha monopolar descartável. $\mathrm{Na}$ porção média, temos como ponto de inserção o meio da linha mediana que vai da escápula ao processo espinhoso do $\mathrm{C}_{7}$, e na porção superior, ângulo entre o pescoço e o ombro.

Para a avaliação dos resultados, classificamos as respostas à estimulação neuromuscular (nervo espinhal/músculo trapézio) em:

1) Normal ou íntegro ( $\mathrm{N}$ ou $\mathrm{I})$.

2) Desnervação parcial (DP): há diminuição do potencial evocado motor e de latência, decorrente da quantidade de fibra nervosa lesada e da tração cirúrgica do nervo. $\mathrm{Na}$ avaliação do músculo trapézio através do eletrodo de agulha, no repouso, surgiram poucas fibrilações e ondas normais. Ao esforço moderado, as fibrilações foram maiores, com alterações das ondas.

3) Reinervação $(R)$ : ocorre o potencial evocado motor com latências dentro de valores normais e boa amplitude. Ao eletrodo de agulha, observou-se silêncio mioelétrico. No esforço moderado, potenciais de ação das unidades motoras normais e no máximo padrão máximo de titulação.

O estudo estatístico foi feito através do teste binominal para avaliação das variáveis analisadas (Essex-Sorlie, 1995), e o Qui-quadrado nas tabelas 2 × 3 para avaliação da associação entre as variáveis, como o tipo de esvaziamento cervical, desnervação parcial, reinervação, radioterapia e idade (significantes para $\mathrm{p}<0,05$ ).

\section{RESULTADOS}

Dos 20 pacientes com carcinoma epidermóide de vias aerodigestivas superiores, 19 eram $\mathrm{N}_{0}$ e $1 \mathrm{~N}_{1}$. Destes, nove foram $\mathrm{pN}_{0}$ e $11 \mathrm{pN}^{+}$. Para os cinco pacientes portadores de neoplasias da tireóide, todos eram portadores de linfonodos clínicos $\left(\mathrm{N}^{+}\right)$e histopatológico positivos $\left(\mathrm{pN}^{+}\right)$. Quando ocorreu mais de um linfonodo metastático, optou-se pela radioterapia em 15 pacientes $(60 \%)$ nas doses de 5 a 6.000cGy. $\mathrm{Na}$ avaliação eletroneuromiográfica da atividade do nervo espinhal e da função do músculo trapézio, em dois pacientes $(6,1 \%)$ as respostas foram normais $(\mathrm{N})$ e em $31(93,9 \%)$ foi diagnosticado DP para $\mathrm{p}<0,001$ (significante por ser $<5 \%$ ). A avaliação do grau de DP nos 31 esvaziamentos, foi moderada em dez $(32,3 \%)$ e severa em $21(67,7 \%)-($ Tabela 2$)$

Tabela 2

Grau de desnervação parcial (DP)

\begin{tabular}{l|c|c}
\hline$D P$ & $N$ & $P$ \\
\hline Moderada & $10(32,3 \%)$ & \\
\hline Severa & $21(67,7 \%)$ & \\
\hline Total & $31(100,0 \%)$ & $<0,001^{*}$ \\
\hline
\end{tabular}

*p significante quando $<5 \%$

Para avaliar a capacidade de reinervação $(\mathrm{R})$ pós-desnervação parcial (DP), esta foi avaliada em 29 pacientes, sendo boa em seis $(21,4 \%)$, regular em $21(71,7 \%)$ e ruim em dois $(7,1 \%)$ (Tabela 3$)$.

Tabela 3

Grau de reinervação $(\mathrm{R})$

\begin{tabular}{l|cc|c}
\hline$R$ & \multicolumn{1}{|c|}{$N$} & $P$ \\
\hline Boa & 6 & $(21,4 \%)$ & \\
Regular & 21 & $(71,5 \%)$ & \\
Ruim & 2 & $(7,1 \%)$ & \\
\hline Total & $29(100,0 \%)$ & $<0,001^{*}$ \\
\hline
\end{tabular}

$*$ p significante quando $<5 \%$

Quanto à relação entre o grau de desnervação parcial (DP) e o tipo de esvaziamento cervical, não houve diferenças significantes - (Tabela 4).

Tabela 4

Relação entre o grau de DP e o tipo de esvaziamento cervical (EC)

\begin{tabular}{|c|c|c|c|}
\hline \multirow[b]{2}{*}{$E C$} & \multicolumn{2}{|c|}{$D P$} & \multirow[b]{2}{*}{$p$} \\
\hline & Moderada & Severa & \\
\hline Supra-omohióideo & $1 \quad(10,0 \%)$ & $5 \quad(23,8 \%)$ & \\
\hline Campo alargado & $2(20,0 \%)$ & $8 \quad(38,1 \%)$ & \\
\hline Funcional & $7 \quad(70,0 \%)$ & $8 \quad(38,1 \%)$ & \\
\hline Total & $10(100,0 \%)$ & $21(100,0 \%)$ & $\mathrm{p}=0,24 \mathrm{C}$ \\
\hline
\end{tabular}

*p não significante 
No que diz respeito aos efeitos das irradiações sobre a reinervação $(\mathrm{R})$, observamos ausência de significância estatística (Tabela 5).

Tabela 5

Relação entre radioterapia (RxT) e reinervação (R)

\begin{tabular}{|c|c|c|c|}
\hline \multirow[b]{2}{*}{$R$} & \multicolumn{2}{|c|}{$R x T$} & \multirow[b]{2}{*}{$p$} \\
\hline & Sim & Não & \\
\hline Regular & $11(73,0 \%)$ & $9 \quad(69,0 \%)$ & \\
\hline Boa & $3(20,0 \%)$ & $3 \quad(23,0 \%)$ & \\
\hline Ruim & $1 \quad(7,0 \%)$ & $1 \quad(8,0 \%)$ & \\
\hline Total & $15(100,0 \%)$ & $13(100,0 \%)$ & 0,97 \\
\hline
\end{tabular}

*p não significante

Quanto à relação entre a idade e a reinervação, 180 dias após a cirurgia foi aferida, como visto na Tabela 6 , sem significância estatística:

Tabela 6

Reinervação ( $\mathrm{R})$ versus idade

\begin{tabular}{|c|c|c|c|}
\hline \multirow{2}{*}{$\begin{array}{l}\text { Reinervação } \\
(R)\end{array}$} & \multicolumn{2}{|c|}{ Idade } & \multirow[b]{2}{*}{$p$} \\
\hline & $\geq 40$ anos & $<40$ anos & \\
\hline Regular & $17(77,0 \%)$ & $3 \quad(50,0 \%)$ & \\
\hline Boa & $4(18,0 \%)$ & $2(33,0 \%)$ & \\
\hline Ruim & $1 \quad(5,0 \%)$ & $1 \quad(17,0 \%)$ & \\
\hline Total & $22(100,0 \%)$ & $6(100,0 \%)$ & $0,37 *$ \\
\hline
\end{tabular}

* p não significante

\section{DISCUSSÃO}

Após a padronização do esvaziamento cervical radical $^{1}$ (1906), trabalhos posteriores demonstraram que a radicalidade oncológica poderia ser preservada através de procedimentos onde o músculo esternocleidomastóideo, o nervo espinhal e a veia jugular interna fossem conservados, impedindo o aparecimento da síndrome de ombro ${ }^{2}$ pela conservação das duas primeiras estruturas referidas. Foram então discutidos os esvaziamentos conservadores como o funcional, campo alargado e o supra-omohióideo, que progressivamente passaram a substituir o clássico ${ }^{3,4,5}$. Nestes procedimentos, o nervo espinhal é dissecado ao longo de sua extensão cervical, sendo conservada sua integridade através de manobras de tração e individualização, causando uma desvascularização e perda funcional parcial, tendo como iatrogenia graus variados de desnervação parcial do músculo trapézio. Estes achados são determinados por alterações da bainha de mielina e conseqüente lesão do axônio denominada axonotmese ${ }^{8}$. A partir do diagnóstico da perda parcial da função das estruturas neuromusculares causada pelas manobras cirúrgicas, envolvendo o nervo espinhal (tração e desvascularização), ainda que conservadoras, detectamos nos 33 esvaziamentos cervicais desnervação parcial do músculo trapézio (Tabela 2) em 31 $(93,99 \%)$ procedimentos, sendo severa em $21(67,7 \%)$ e moderada em $10(32,3 \%)$, significantes e concordantes com a literatura ${ }^{9,10}$.

Para avaliar a reinervação $(\mathrm{R})$, a capacidade de recuperação do músculo trapézio se faz pela regeneração de até $80 \%$ das fibras nervosas do nervo espinhal lesado (axoniotmese), com crescimento do axônio de $2 \mathrm{~mm} / \mathrm{dia}$. Como este nervo tem de 10 a $15 \mathrm{~cm}$ de comprimento, sua recuperação se faz em quatro meses ${ }^{8}$. Em nossa casuística (Tabela 3), a reinervação foi boa em $21,4 \%$ dos casos, regular em $71,5 \%$ e ruim em $7,1 \%$. Isto permite compreender que a recuperação da unidade neuromuscular depende fundamentalmente das manobras cirúrgicas ${ }^{11,12}$ (trauma cirúrgico, idade do paciente, tropismo muscular e presença ou não de linfonodos metastáticos no triângulo posterior).

No que diz respeito à relação entre o grau de DP e o tipo de esvaziamento cervical, não ocorreram diferenças significantes (Tabela 4) que justifiquem a opção por um deles, visto que as alterações incidiram em todos os três procedimentos empregados, concordantes com a literatu$\mathrm{ra}^{13}$. Já as variáveis radioterapia (Tabela 5) e a idade (Tabela 6) não foram responsáveis pelas alterações detectadas na unidade neuromuscular. Em síntese, concluímos que a ENM constitui-se num método efetivo na avaliação da unidade neuromuscular (nervo espinhal/músculo trapézio) e que o tipo de esvaziamento cervical conservador não foi determinante de alterações relevantes na avaliação da casuística.

\begin{abstract}
Background: Twenty fire patients with malignant head and neck neoplasias (20 from superior aerodigestive tract and 5 from thyroid gland), were submitted to 33 unilateral or bilateral neck node dissection (15 supraomohyoid, 11 modified and 7 wide field), at the Head and Neck Service of Hospital Araújo Jorge, Goiânia, Goiás, Brazil. Method: Eletroneuromiography (ENM) of the trapezius muscle after spinal nerve dissection. Results: In spite of surgical dissection, 94\% of trapezius muscle desnervation were detected, being $38 \%$ severe and $62 \%$ moderate, $(p=0,001)$. The degree of lesion for spinal nerve after 30 days, showed axoniumtmese in 31 of 33 procedures, and after 180 days, $21 \%$ of severe, $72 \%$ of moderate and $7 \%$ of unsatisfactory reinervation were diagnosed $(p=0,001)$. Conclusions: ENM is an effective method of evaluation of neuromuscular unit (trapezius muscle/spinal nerve) and the type of neck node dissection was not determinant of structural nerve alteration.
\end{abstract}

Key Words: Trapezius Muscle; Spinal Nerve; Neck Dissection; Eletroneuromiography. 


\section{REFERÊNCIAS}

1. Crile G. Excision of cancer of the head and neck. JAMA, 1906; 47:1780-1786.

2. Ewing M, Martin. Disabillity following "radical neck dissection". Cancer, 1952; 5:873-883.

3. Nahum A, Mullally W, Marmor LA. Syndrome resulting from radical neck dissection. Arch Otolaryngol, 1961; $74: 424-428$.

4. Suarez O. El problema de las metastasis linfaticas y alejadas del cancer de laringe e hipofaringe. Rev Otorhinolaryngol, 1963; 23: 83-99.

5. Bocca E, Pignataro O, Sasaki CT. Functional neck dissection. A description of operative technique. Arch Otolaryngol, 1980; 106: 524-527.

6. Ballantyne AJ, Guinn GA. Reduction of shoulder disability after neck dissection. Ann Surg, 1966; 113: 662-665.

7. Galvani. Apud Pinto LC. Eletroneuromiografia Clínica. $1^{a}$ Ed. Guanabara Koogan; 1996; 29.

8. Seddon HJ. Three types of nerve injury. Brain, 1943; 66: 237-286.

9. Andrade Sobrinho J, Fava AS, Menezes RM et al. Neurorrafia do nervo espinal acessório após esvaziamento cervical radical para reabilitação funcional do ombro. Rev Col Bras Cir; 1992, 19: 16-20.

10. Koybasioglu A, Tokcaer AB, Uslu SS et al. Acessory nerve function after modified radical and lateral neck dissection. Laryngoscope, 2000; 110: 73-77.

11. Krause HR, Bremerich A, Hermann M. The innervation of the trapezius muscle in conection with radical neck dissection. J Craniomaxillofac Surg, 1983; 146: 526530.
12. Petrera J, Troyaborg W. Conduction studies along the accessory nerve and follow-up of patients with trapezius palsy. J Neur Neurosur Psych, 1983; 47: 630-636.

13. Schuller DE, Hamarker RC, Lingeman RE et al. Analysis of disability resulting from treatment incluind radical neck dissection or modified neck dissection. Head Neck Surg, $1983 ; 6: 551-558$.

Endereço para correspondência:

Prof. Dr. Abrão Rapoport

Pça. Amadeu Amaral, 47 - cj. 82 - Paraíso

01450-020 — São Paulo-SP

E-mail: cpgcp.hosphel@attglobal.net 